\section{Impact of voxelotor (GBT440) on unconjugated bilirubin and jaundice in sickle cell disease}

\author{
Paul Telfer, ${ }^{1}$ Irene Agodoa, ${ }^{2}$ \\ Kathleen M. Fox, ${ }^{3}$ Laurie Burke, ${ }^{3}$ \\ Timothy Mant, ${ }^{4}$ Marzena Jurek, ${ }^{2}$ \\ Margaret Tonda, ${ }^{2}$ Josh Lehrer-Graiwer ${ }^{2}$ \\ ${ }^{1}$ Centre for Genomics and Child Health, \\ Blizard Institute, Barts and The London \\ School of Medicine and Dentistry, \\ Queen Mary University of London, UK; \\ ${ }^{2}$ GBT, South San Francisco, CA, USA; \\ ${ }^{3}$ LORA Group, Royal Oak, MD, USA; \\ ${ }^{4}$ IQVIA, Reading, UK
}

\begin{abstract}
For many patients with sickle cell disease (SCD), jaundice is a significant clinical disease manifestation that impacts on patient well-being. We report a case of a patient with SCD and chronic jaundice treated with voxelotor (GBT440), a novel small molecule hemoglobin oxygen affinity modulator and potential disease-modifying therapy for SCD. The case patient is a $27-$ year-old Black male with a long history of SCD with clinical jaundice and scleral icterus. After starting voxelotor, the patient reported that his jaundice cleared within one week, and that he felt much better with more energy, and was relieved after his eyes cleared. Voxelotor reduced bilirubin and unconjugated bilirubin (by up to $76 \%$ ), and hemoglobin improved from $9.9 \mathrm{~g} / \mathrm{dL}$ at baseline to $11.1 \mathrm{~g} / \mathrm{dL}$ at 90 days. Jaundice impacts many adults with SCD, significantly impacting self-image. Voxelotor treatment reduced bilirubin levels and improved jaundice, resulting in an improved sense of well-being in our case patient.
\end{abstract}

\section{Introduction}

Sickle cell disease (SCD), an inherited chronic hematological disorder affecting millions worldwide, causes significant morbidity and reduced life expectancy. ${ }^{1}$ For many patients with SCD, jaundice is a significant clinical manifestation which impacts patient well-being and quality of life. ${ }^{2}$ We report a case of a patient with SCD and chronic jaundice treated with voxelotor (GBT440), a novel small molecule hemoglobin oxygen affinity modulator and potential disease-modifying therapy for SCD.

\section{Case Report}

The patient is a 27-year-old Black male diagnosed with SCD at birth who recalls learning about his SCD from the age of 7 years. In this patient, jaundice has manifested as scleral icterus. Relevant medical history in the previous 12 months included two hospitalizations for painful crises, no blood transfusions, and no hydroxyurea treatment. SCD symptoms included pain, fatigue, yellow eyes, stress, and a feeling of being overwhelmed. Minor crises occurred two to three times per week; the patient noted these as bad days and managed his symptoms at home by taking analgesics and resting.

The patient participated in two voxelotor trials; a phase 1 study (NCT02285088) in which he received placebo, and a phase 2 follow-on extension (NCT03041909) in which he received voxelotor $900 \mathrm{mg}$ orally once daily for six months. Liver function test values at baseline and end of study, respectively, were as follows: alkaline phosphatase, 57 and 62 IU/L (normal range values, 30-130 IU/L); alanine aminotransferase, 30 and $24 \mathrm{U} / \mathrm{L}$ (normal value, $<45$ IU/L); and aspartate aminotransferase, 52 and $35 \mathrm{U} / \mathrm{L}$ (normal value, $<45 \mathrm{IU} / \mathrm{L}$ ). Total and unconjugated bilirubin at baseline were 112 and $101 \mu \mathrm{mol} / \mathrm{L}$ (6.5 and $5.9 \mathrm{mg} / \mathrm{dL})$, respectively (normal range values, 3-22 $\mu \mathrm{mol} / \mathrm{L}$ or $0.2-1.3 \mathrm{mg} / \mathrm{dL}$ [total bilirubin] and $3-17 \mu \mathrm{mol} / \mathrm{L}$ or $0.2-1.0 \mathrm{mg} / \mathrm{dL}$ [unconjugated bilirubin]), and hemoglobin was 9.9 $\mathrm{g} / \mathrm{dL}$ (normal range values, 14-18 g/dL [men] and 12-16 g/dL [women]). During the follow-on trial, voxelotor treatment reduced bilirubin over time (Figure 1); unconjugated bilirubin was reduced by up to $76 \%$ (Table 1). Hemoglobin improved from $9.9 \mathrm{~g} / \mathrm{dL}$ at baseline to $11.1 \mathrm{~g} / \mathrm{dL}$ at 90 days.

A structured 60-minute in-depth interview was conducted on June 3, 2017 to provide the patient's description and experience with jaundice before, during, and after voxelotor treatment. The patient's subjective experience of his chronic scleral icterus mirrors the pattern of change in his laboratory values and highlights the social stigma associated with this clinical symptom of disease. Before voxelotor treatment, the patient reported that his eyes had been yellow or yellow-greenish since he was young, and that it was an everyday thing for me, leading him to wear tinted glasses. His appearance was a source of emotional distress, particularly at a young age. Within one week of starting voxelotor, the patient's eyes were back to normal with no sign of yellow and stayed white throughout the
Correspondence: Paul Telfer, Department of Haematology Barts Health NHS Trust, Royal London Hospital, Whitechapel Road London E1 1BB UK.

E-mail: paul.telfer@bartshealth.nhs.uk

Key words: sickle-cell disease, jaundice, scleral icterus, voxelotor.

Acknowledgments: The authors would like to acknowledge Rick Davis, whose work was funded by GBT, for writing assistance, and Noel Landsman for his assistance with data collection.

Contributions: the authors contributed equally.

Conflict of interest: Paul Telfer: ApoPharma and Terumo (honoraria); Bluebird Bio, Novartis, and GBT (consultancy); Kyora Kirin and Mundi Pharma (research funding); Pfizer (other, participation in EDMC). Irene Agodoa, Marzena Jurek, Margaret Tonda, and Josh Lehrer-Graiwer: GBT (employment and equity ownership); Kathleen M. Fox and Laurie Burke: GBT. (consultancy); Timothy Mant: IQVIA (employment and research funding); NIHR (research funding).

Received for publication: 23 February 2018 Revision received: 21 May 2018.

Accepted for publication: 21 May 2018.

This work is licensed under a Creative Commons Attribution-NonCommercial 4.0 International License (CC BY-NC 4.0).

CCopyright P. Telfer et al., 2018

Licensee PAGEPress, Italy

Hematology Reports 2018; 10:7643

doi:10.4081/hr.2018.7643

trial, which allowed him to stop wearing tinted glasses. Friends and family noticed the change in his eyes. The patient reported that he had more energy and felt stronger, and the minor crises stopped. He also stated that he exercised, ate, and drank more, and also perceived a sense of delight, much happier, great feeling. He indicated that since birth, nothing has made my eyes go clear; I tried so many different things and they didn't help and within a short time on the medication, my eyes were clear.

After stopping voxelotor, the patient's eyes rapidly returned to their previous yellow color, which persisted on a daily and chronic basis. While receiving voxelotor, the patient felt so much better; the SCD pain was gone; felt happier, took away the stress and worrying about pain, and now felt worse while off the study medication. His friends noted that during the trial that he 
looked healthier. Other benefits described by the patient while receiving voxelotor included relief of pain, less stress, and better sense of well-being. These benefits dissipated after voxelotor discontinuation. The patient rated the study medication as very beneficial to his overall quality of life.

\section{Discussion}

Jaundice is a common sign/symptom in SCD and a common presenting symptom in adults with acute sickle cell syndromes. ${ }^{3}$ The accelerated breakdown of sickled red blood cells in SCD results in increased circulating levels of bilirubin, which is responsible for yellowing of the skin and eyes in patients with SCD. ${ }^{4}$ Jaundice has been reported in up to $84 \%$ of patients with SCD in clinical studies, ${ }^{5-7}$ and the proportion of SCD patients with jaundice appears to rise with age. ${ }^{8}$ Jaundice can also impact selfimage. In a cross-sectional survey of 100 SCD patients, 79\% reported having jaundice, $69 \%$ reported that others mentioned their jaundice, $39 \%$ were bothered by their jaundice, and $37 \%$ noted being uncomfortable when others asked about their jaundice. ${ }^{2}$ In addition, total bilirubin was positively correlated with personal, relational, and behavioral survey subscales. These results suggest that jaundice significantly impacted the self-image of respondents. Moreover, beyond the embarrassment associated with jaundice that is bothersome to patients and lowers their sense of well-being, patients may use their eye yellowness to judge their disease severity. Indeed, our case patient worried about worsening of symptoms when he observed a darker yellow eye color, and said he was constantly reminded of his disease when his eyes were yellow, which may also have negatively impacted his sense of wellbeing.

Information from social media sites also suggests that scleral icterus is an important issue for patients with SCD. ${ }^{9}$ Patients indicate that people notice and comment on their yellow eyes, that they feel embarrassed and despondent, and that it affects their personal and professional lives. Lastly, there are many queries on these social media sites asking about available drugs to treat their jaundice. This information indicates that scleral icterus is an underappreciated issue and that additional SCD treatment options are needed.

Voxelotor is an investigational novel small molecule hemoglobin oxygen affinity modulator and a potent anti-sickling agent that dose-dependently increases hemoglo- bin, hematocrit, and erythrocyte counts and reduces unconjugated bilirubin and reticulocytes in patients with SCD. ${ }^{10-12}$ Pooled interim results from the phase 1 study and phase 2 extension study in 41 SCD patients who received up to six months' treatment with voxelotor $500-1000 \mathrm{mg}$ /day showed hematologic response in all patients, including marked reduction in hemolysis and peripheral blood sickle cells and a clinically significant increase in hemoglobin $(46 \%$ with $>1 \mathrm{~g} / \mathrm{dL}$ increase $v s 0 \%$ for placebo; $\mathrm{P}=0.006) .{ }^{10}$ Median unconjugated bilirubin was reduced by $40 \%$. No serious or severe adverse events related to study treatment were reported with up to six months' dosing of voxelotor (all treatment-related adverse events were grade 1 or 2), and no sickle cell crisis events or evidence of hypoxia were observed in any patients on study drug.

The decline in unconjugated bilirubin in our case patient is consistent with that previously reported (median decrease of $43 \%$ at the $900 \mathrm{mg} / \mathrm{d} \operatorname{dose}^{10}$ ), and was below the bilirubin threshold typically associated with clinical jaundice $(3 \times$ upper limit of normal, $51 \mu \mathrm{mol} / \mathrm{L}$ ) for most of the treatment period. In study patients who received voxelotor for $\geq 90$ days, there were two additional patients with bilirubin elevated to the range of jaundice at baseline who had similar resolution of hyperbilirubinemia.

Table 1. Unconjugated bilirubin and change from baseline over time with voxelotor treatment.

\begin{tabular}{lcc}
\hline Study day & Unconjugated bilirubin, $\mathrm{Jmol} / \mathrm{L}$ & Change from baseline, \% \\
0 (Enrolment) & 101 & - \\
15 & 62 & -39 \\
\hline 30 & 24 & -76 \\
45 & 42 & -58 \\
\hline 60 & 38 & -62 \\
90 & 42 & -58 \\
\hline 120 & 50 & -51 \\
150 & 27 & -73 \\
\hline 180 (end of study treatment) & 46 & -54 \\
208 (28 days after last dose of voxelotor) & 82 & -19 \\
\hline
\end{tabular}

Normal range values are $3-17 \mu \mathrm{mol} / \mathrm{L}(0.2-1.0 \mathrm{mg} / \mathrm{dL})$ for unconjugated bilirubin.

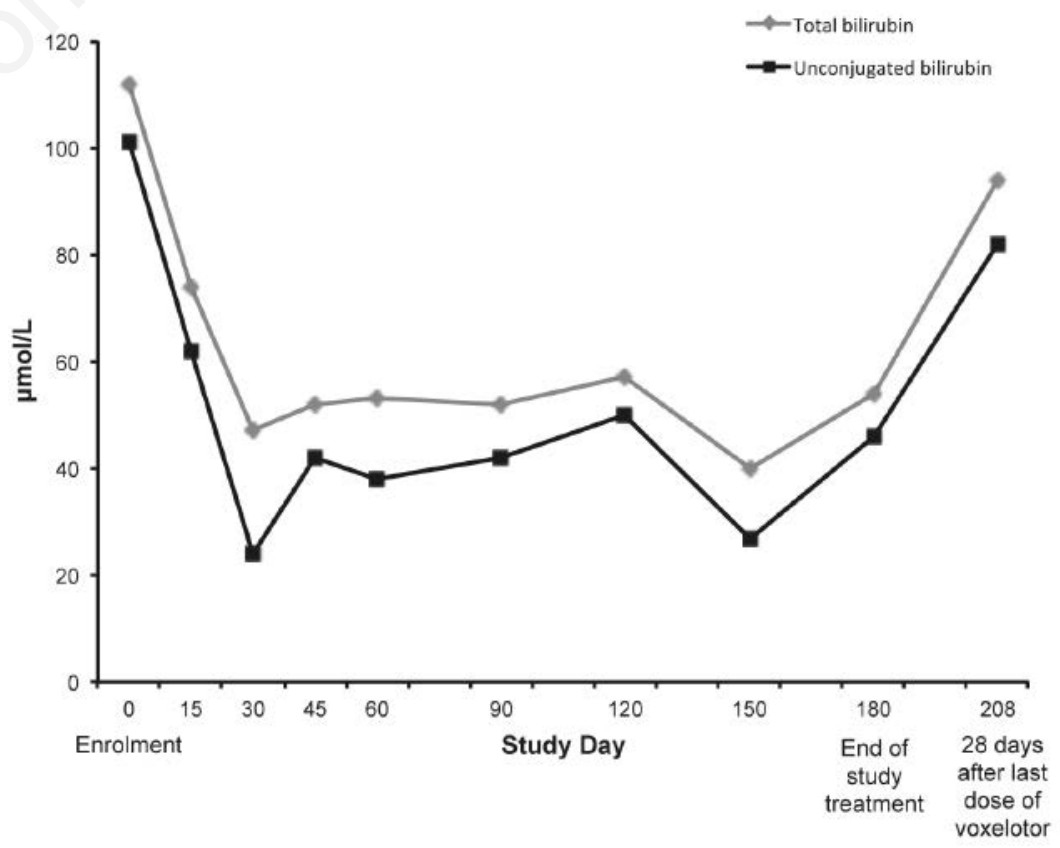

Figure 1. Total and unconjugated bilirubin over time with voxelotor treatment. Normal range values are 3-22 $\mu \mathrm{mol} / \mathrm{L}(0.2-1.3 \mathrm{mg} / \mathrm{dL})$ for total bilirubin and 3-17 $\mu \mathrm{mol} / \mathrm{L}(0.2-$ $1.0 \mathrm{mg} / \mathrm{dL}$ ) for unconjugated bilirubin. 


\section{Conclusions}

The case patient had a long history of SCD with clinical jaundice that manifested as scleral icterus. The patient's jaundice cleared within 1 week of starting voxelotor and he felt much better with more energy, and was relieved/very happy after his eyes cleared. The jaundice returned after the patient stopped voxelotor. Jaundice affects many adults with SCD, significantly impacting self-image. Among the potential benefits associated with reduction of red blood cell hemolysis through prevention of sickle hemoglobin polymerization, this case report showed that voxelotor treatment reduced bilirubin levels and improved jaundice, resulting in an improved sense of wellbeing.

\section{References}

1. Piel FB, Steinberg MH, Rees DC. Sickle cell disease. N Engl J Med
2017;376:1561-73.

2. Nero AC, McCavit TL, Adix LM, et al. Impact of jaundice on adults with sickle cell anemia. Blood 2012;120:4753.

3. Kaur M, Das GP, Verma IC. Sickle cell trait and disease among tribal communities in Orissa, Madhya Pradesh \& Kerala. Indian J Med Res 1997;105: 111-6.

4. Ilesanmi OO. Pathological basis of symptoms and crises in sickle cell disorder: implications for counseling and psychotherapy. Hematol Rep 2010;2:e2.

5. Maher MM, Mansour AH. Study of chronic hepatopathy in patients with sickle cell disease. Gastroenterol Res 2009;2:338-43.

6. Kumar R, Panigrahi I, Dalal A, Agarwal S. Sickle cell anemia-molecular diagnosis and prenatal counseling: SGPGI experience. Indian J Pediatr 2012;79: 68-74.

7. Karayalcin G, Rosner F, Kim KY, et al. Sickle cell anemia- clinical manifestations in 100 patients and review of the literature. Am J Med Sci 1975;269:5168.
8. de Montalembert M, Guilloud-Bataille M, Feingold J, Girot R. Epidemiological and clinical study of sickle cell disease in France, French Guiana and Algeria. Eur J Haematol 1993;51:136-40.

9. Sickle Cell Warrior. Yellow eyes and sickle cell. Available at: http://sicklecellwarriors.com/yellow-eyes-and-sicklecell/\#comments Accessed Sept 8, 2017.

10. Howard J, Hemmaway CJ, Telfer P, et al. Long-term dosing in sickle cell disease subjects with GBT440, a novel $\mathrm{HbS}$ polymerization inhibitor. 58th Annual Meeting of the American Society of Hematology. San Diego, CA; 2016.

11. Dufu K, Lehrer-Graiwer J, Ramos E, Oksenberg D. GBT440 inhibits sickling of sickle cell trait blood under in vitro conditions mimicking strenuous exercise. Hematol Rep 2016;8:6637.

12. Lehrer-Graiwer J, Howard J, Hemmaway CJ, et al. Long-term dosing in sickle cell disease subjects with GBT440, a novel HbS polymerization inhibitor. Blood 2016;128:2488. 